\title{
Effect of lifestyle modifications on prediabetes
}

\author{
Shreya Srugaram ${ }^{1}$ and Robert Zynda\#
}

${ }^{1}$ Troy High School, Troy, MI, USA

\#Advisor

\section{$\underline{\text { ABSTRACT }}$}

The emergence of non-insulin dependent diabetes mellitus (NIDDM) as a major health issue over the past few decades has made it necessary to set up protocols to prevent or defer its onset especially in genetically predisposed individuals. Prediabetic state has become a very common condition that indicates a blood sugar level higher than the normal levels in the human body. More than 100 million Americans has prediabetes or diabetes ${ }^{[1]}$. Without any form of intervention, $37 \%$ of the prediabetic individuals will develop diabetes in 4 years. Prediabetes is now recognized as a reversible condition. Hence, increased awareness and lifestyle interventions in terms of exercise and dietary modifications seem to be of much benefit for this group to help prevent a largely preventable disease. This study which was conducted over a period of 6 months is designed to explore the role of beneficial lifestyle modifications in prediabetics.

\section{Introduction}

During the past decade, non-insulin dependent diabetes mellitus (NIDDM) has emerged as an important clinical and health problem. The global and regional statistics provided by the International Diabetes Federation shows the increase in diabetes is a major concern. World Diabetes Day is held every year on November $14^{\text {th }}$ to spread awareness on diabetes mellitus. Prediabetes is defined as a condition in which people have higher than normal blood sugar levels but not high enough for the diagnosis of diabetes. According to the American Diabetes Association (ADA), the diagnostic criteria for prediabetes is an elevated fasting plasma glucose level of $100 \mathrm{mg} / \mathrm{dL}-125 \mathrm{mg} / \mathrm{dL}$ or a glycated hemoglobin (HbA1c) value of $5.7 \%$ to $6.4 \%$ or an elevated plasma glucose level after an oral glucose tolerance test $(140 \mathrm{mg} / \mathrm{dL}-199 \mathrm{mg} / \mathrm{dL})$. HbA1c test provides an average of 3 months blood glucose level. The HbA1c level 6.5 or greater is considered diabetic ${ }^{[2]}$.

ADA diagnostic criteria for normal, prediabetics and diabetics

\begin{tabular}{|l|l|l|l|}
\hline Diabetes Test & Normal & Prediabetes & Diabetes \\
\hline Hemoglobin A1c & $<5.7$ & $5.7-6.4$ & $>6.5$ \\
\hline $\begin{array}{l}\text { Fasting Blood Glucose } \\
\mathrm{mg} / \mathrm{dL}\end{array}$ & $<100$ & $100-125$ & $>125$ \\
\hline $\begin{array}{l}\text { Oral Glucose Toler- } \\
\text { ance Test mg/dL }\end{array}$ & $<140$ & $140-199$ & $>199$ \\
\hline
\end{tabular}

Prediabetes, now recognized as a reversible condition, increases an individual's risk for development of diabetes. Lifestyle risk factors for prediabetes include inappropriate eating habits, having a higher body mass index (BMI) than normal and physical inactivity. Several benefits to people having genetic predisposition for diabetes have been noted with lifestyle changes such as healthy eating along with daily exercise and with regular screening ${ }^{[3]}$. The test of glycated hemoglobin (H1bA1c) at least once a year to earmark the people likely to develop the condition may be of 
assistance to delay its onset. In this study, a plan framed with diet modification and exercise along 3 monthly tests of H1bA1c to check the effect may be helpful.

\section{Aim of the Study}

Lifestyle changes which include a diet plan and daily exercise of 30 minutes for people with deranged HbA1c levels in the prediabetic range with a family history of NIDDM can be made so that the onset of disease can be deferred or prevented.

\section{Material and Method}

The study was conducted over a period of six months from $05 / 15 / 2021$ to $10 / 24 / 2021$. The members of 14 families were included in the study after taking their consent. The sample size of the study was 28 . The inclusion criteria were

- $\quad$ Age Group: 31 to 42 years.

- Positive family history of NIDDM

- Normal BMI

- HbA1c levels in the prediabetic range

A 3-monthly follow-up of HbA1c was conducted. HbA1c values were checked at the start of the study and then after 3 months and 6 months. The fasting blood sugar levels were measured daily with a glucometer. The test was done only with a glucometer because there is little variation of levels between the glucometer values and laboratory test values. The glucometer checks the capillary blood sugar levels, and the laboratory checks venous blood sugar levels. The capillary blood sugar level is a little higher than the venous blood sugar level as complete utilization of sugar in tissue is still pending ${ }^{[4]}$. A diet chart comprising of mostly low glycemic index (GI) food items was designed to find out the variation of daily blood sugar levels ${ }^{[5]}$. An attempt is made to keep it low and steady. The value of dietary supplementation of fenugreek, in the form of fenugreek seeds, was also accessed ${ }^{[6]}$. Lifestyle modifications apart from dietary changes also included an introduction of daily exercise for 30 minutes in the form of brisk walking.

\section{Observation and Analysis}

\section{Observation I (age distribution)}

Number of study subjects - 28. Age distribution of subjects was noted. 


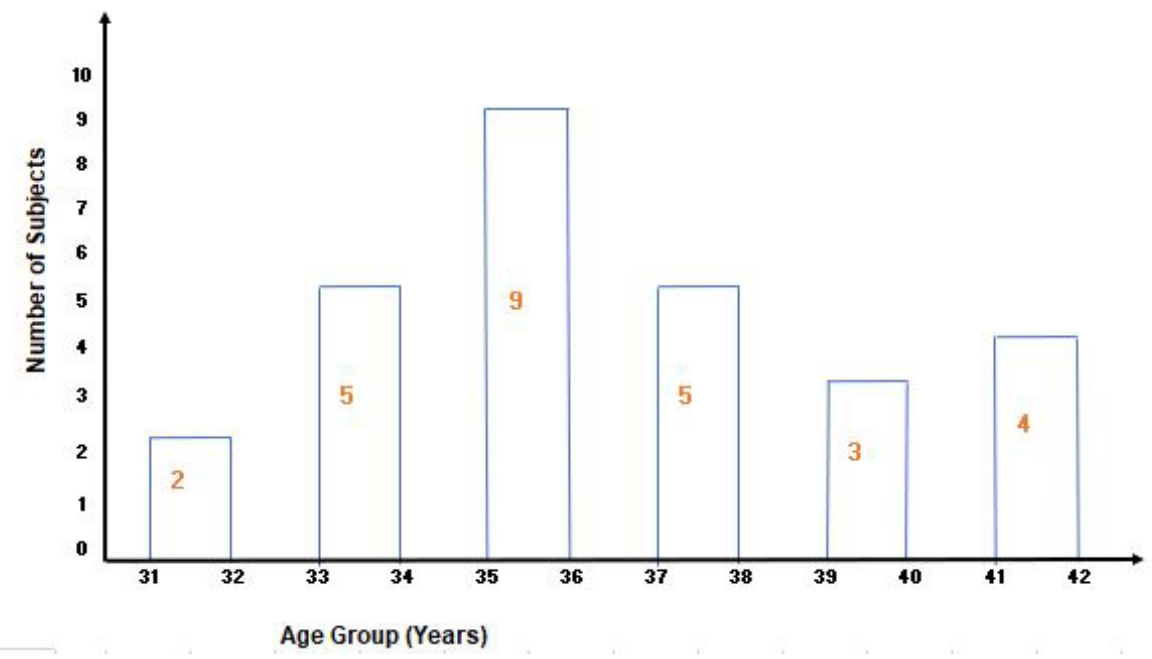

Fig 1. Details of the age distribution of subjects

In the study, the prevalence of deranged HbA1c was found highest in the age group of 35 to 36 years.

\section{Observation II (family history)}

Family history of subjects was noted in reference to diabetes mellitus

Group A consists of number of people with one parent diabetic - 21

Group B consists of number of people with both parent diabetic -7

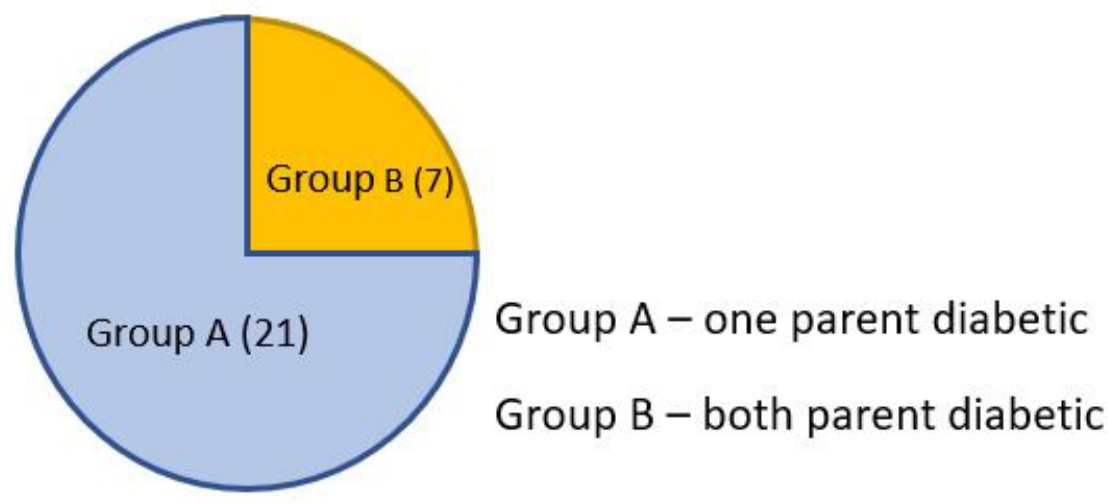

Fig 2. Pie chart showing the family history of subjects in relation to diabetes mellitus (NIDDM)

On comparing with values of HbA1c of subjects in Group A and Group B no significant difference was found.

Observation III (initial HbA1c values)

Subjects were randomly divided into 3 categories after their consent and taking their wishes into consideration. 
Category I consists of 7 subjects, with no change in diet and lifestyle (Control)

Category II consists of 14 subjects, who were started on diabetic diet consisting of low Glycemic Index (GI) food along with 30 minutes daily exercise.

Category III consists of 7 subjects, along with a diabetic diet and 30 minute daily exercise, they also consumed fenugreek seeds (1 teaspoon full soaked overnight).

Subjects from Group A and Group B (from observation 2) were considered together as their level of initial HbA1c did not show much variation.

The HbA1c values of all test subjects were recorded at the initiation of the study.

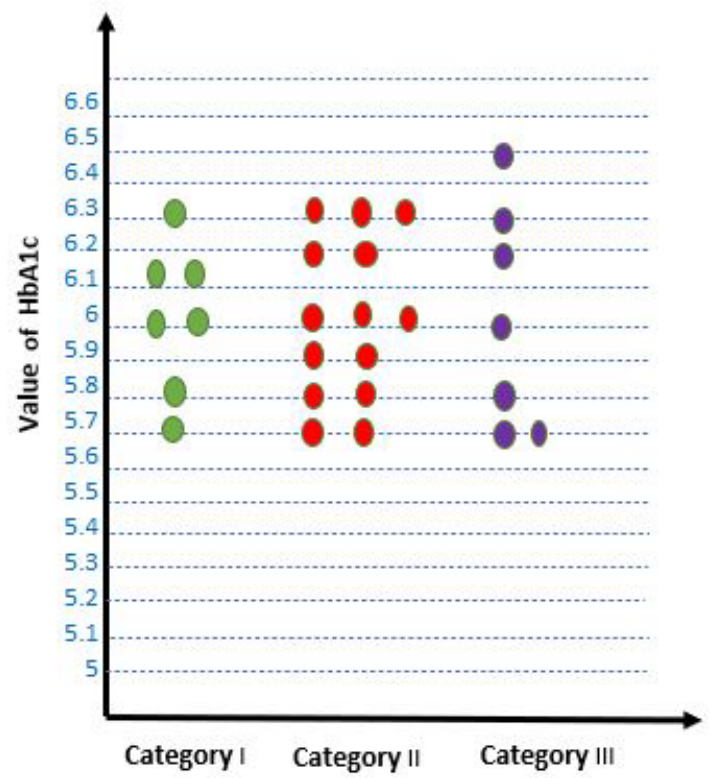

Fig 3. Scattergram showing HbA1c levels of test subjects at the beginning of the study

Observation IV (lifestyle changes - daily fasting blood sugar values)

The daily fasting blood sugar values were self-recorded by the participants with the help of a glucometer in Category I, Category II, and Category III. Their average blood sugar value was calculated category-wise at monthly intervals for 6 months. 


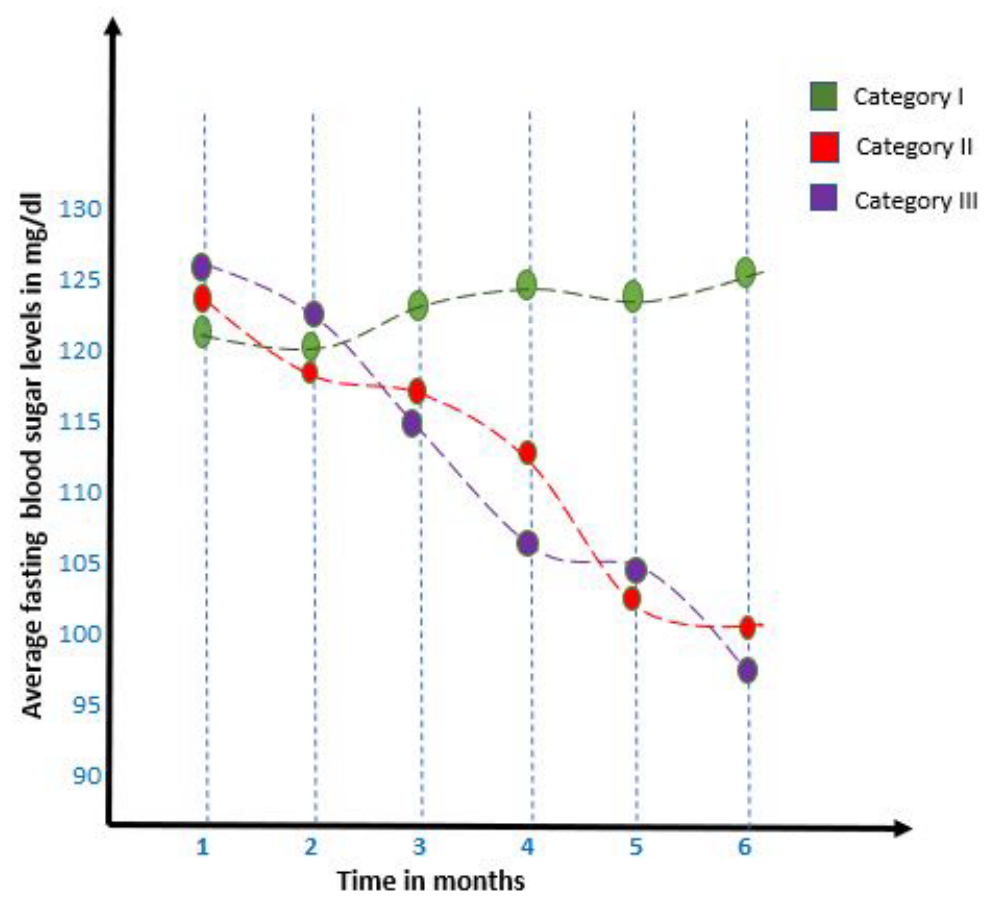

Fig 4. Line graph showing average fasting blood sugar values over a period of 6 months

In category I, no decline instead a small rise in the average fasting blood sugar values was noted. In category II and category III, a very gradual but steady decline of fasting blood sugar values was observed. Not much difference in category II and category III values was observed.

Multiple studies have evaluated the potential anti-diabetic benefits of fenugreek seeds. Fenugreek seeds are high in soluble fibers which help lower blood sugar levels by slowing down digestion and absorption of carbohydrates. However, in the study, we did not find a significant difference in category II and category III values.

Observation V (lifestyle changes - HbA1c values after 3 months and at the end of 6 months)

The HbA1c values were measured after the end of 3 months and at the end of 6 months, to see the effect of proposed lifestyle changes 


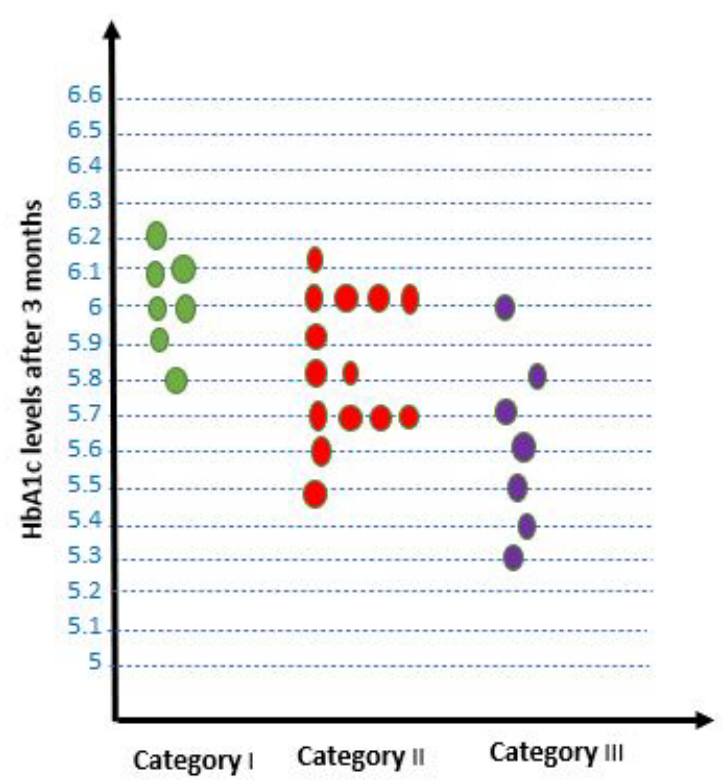

Fig 5. Scattergram showing HbAlc values after 3 months

Estimation of $\mathrm{HbA1c}$ levels at the end of 3 months shows that participants in category I did not show any improvement in HbA1c levels. Most participants in category II and category III showed improvement in HbA1c levels at the end of 3 months but not all indicating that there are individual variations among people for similar carbohydrate load.

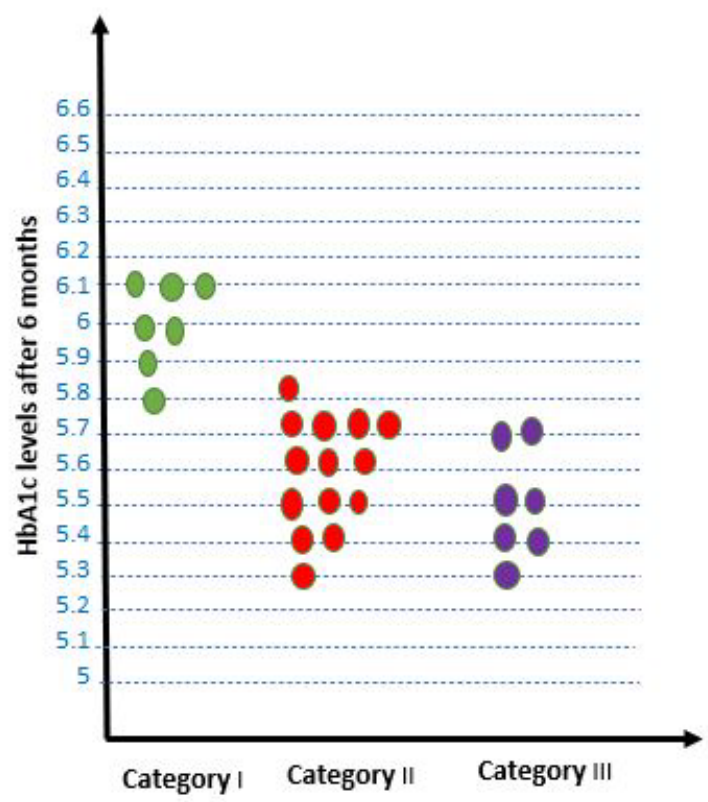

Fig 6. Scattergram showing $\mathrm{HbA} 1 \mathrm{c}$ values after 6 months 
Estimation of HbA1c levels at the end of 6 months shows that compared to category I (control) there was a marked reduction in HbA1c levels in category II and category III participants. Although there is an individual variation with the same diet and exercise regimen, a long time following of dietary regimen along with lifestyle changes is of help in lowering HbA1c levels and achieving good glycemic control.

\section{Conclusion}

An attempt has been made through this study to highlight the importance of lifestyle interventions in terms of dietary changes and inclusion of exercise in dietary routine. The study shows that food with a low glycemic index (GI) is of importance especially for people having a genetic disposition for diabetes. Dietary planning for prediabetic people is helpful in controlling blood sugar levels. Meticulous individualized planning of dietary charts is essential for maximum benefit as there can be individual variations among people for similar carbohydrate load. Long-term data from various studies suggest that lifestyle interventions may decrease the risk of progression from prediabetes to diabetes for as long as 10 years. Hence, with such interventions, prediabetes can be controlled and even reversed.

\section{Limitations}

The study was performed with a sample size of 28, and the age group range was 31 years to 42 years. The study can be further done with a larger sample size. A study with a larger sample size can be done and results can be compared to affirm the conclusions. The age limitations can be broadened, and research can be conducted to yield a valuable conclusion relating to prediabetes control and reversal with the age factor on a broader scale.

\section{Acknowledgments}

My sincere thanks to my teacher Robert Zynda, Troy High School Michigan for his valuable guidance and support. I express my deep gratitude to Dr. Dipanwita Dutta, Senior Consultant, Department of Obstetrics and Gynecology, Apollo Cradle Hospital, Indrapuram, India, whose valuable guidance, constant attention, and sincere efforts were constantly at disposal throughout the present work. I would like to express my sincere thanks to my family for their support. Last but not the least, I am grateful to all whose valuable knowledge was utilized in this work.

\section{References}

1. National Diabetes Statistics Report, 2020 | CDC

https://www.cdc.gov/diabetes/library/features/diabetes-stat-report.html

2. AmericAmerican Diabetes Association. 2. Classification and Diagnosis of Diabetes: Standards of Medical Care in Diabetes-2021. Diabetes Care. 2021 Jan;44(Suppl 1):S15-S33.

3. Schellenberg ES, Dryden DM, Vandermeer B, Ha C, Korownyk C. Lifestyle interventions for patients with and at risk for type 2 diabetes: a systematic review and meta-analysis. Ann Intern Med. 2013 Oct 15;159(8):54351. DOI: http://dx.doi.org/10.7326/0003-4819-159-8-201310150-00007. [PubMed] [Google Scholar]

4. Juneja D, Pandey R, Singh O. Comparison between arterial and capillary blood glucose monitoring in patients with shock. Eur J Intern Med. 2011;22:241-4. [PubMed] [Google Scholar] 
5. https://www.diabetes.co.uk/diet/glycaemic-index-diet-and-diabetes.html

6. Vijayakumar MV, Singh S, Chhipa RR, Bhat MK. The hypoglycaemic activity of fenugreek seed extract is mediated through the stimulation of an insulin signalling pathway. Br J Pharmacol. 2005;146:41-8.- PMC - PubMed

7. Diabetes research clinical practice

https://www.diabetesresearchclinicalpractice.com/article/S0168-8227(17)30375-3/fulltext 